\title{
Moralização sobre o uso de álcool entre estudante de curso de saúde
}

\author{
Leonardo Fernandes Martins \\ Pollyanna Santos da Silveira \\ Rhaisa Gontijo Soares \\ Henrique Pinto Gomide \\ Telmo Mota Ronzani \\ Universidade Federal de Juiz de Fora
}

\begin{abstract}
Resumo
O uso de álcool é uma das principais condições que apresentam conotação moralizante no mundo. O objetivo deste estudo foi investigar os estereótipos e a atribuição moral de graduandos de cursos da área da saúde acerca da dependência de álcool. Participaram do estudo 138 estudantes matriculados nos cursos de Enfermagem, Medicina, Psicologia e Serviço Social. Os instrumentos utilizados foram: questionário sobre características do estudante, questionário sobre estereótipos e moralização do uso de álcool. Verificou-se que para uso de álcool o modelo de percepção que os estudantes possuíam era o moral e que uma de suas maiores dificuldades era lidar com alcoolistas.
\end{abstract}

Palavras-chave: alcoolismo; estereotipagem; graduação em saúde; atitudes.

\begin{abstract}
Moralization about alcohol use among health college students. Alcohol use is one of the major conditions that display a worldwide moralizing connotation. The objective of this present article was to investigate the stereotypes and the moral attribution of the Health College Students (HCS) about the dependence of alcohol. 138 HCS enrolled on undergraduate health courses participated of the study. Three instruments were used: questionnaire about the personal characteristics of the professionals, Evaluation questionnaire about stereotypes and moralization of the use of alcohol. Was verified that for the use of alcohol, the perception model of the HCS was moral and one of the biggest difficulties for them dealing with the patients referred to the alcoholics.
\end{abstract}

Keywords: alcoholism; stereotyping; undergraduate; health courses; attitudes.

$\mathrm{O}$ consumo de álcool está presente há milhares de anos na história da humanidade, sendo utilizado desde rituais religiosos até práticas recreativas em diversas culturas (Dietler, 2006), entretanto, o seu consumo de forma abusiva pode estar relacionado ao surgimento de mais de sessenta tipos de doenças e agravos diferentes (Rehm et al., 2003), estando também associado a problemas sociais, incluindo problemas familiares e interpessoais (World Health Organization [WHO], 2007). Além dos impactos diretos à saúde, em termos de morbidade e mortalidade dos indivíduos que fazem o consumo abusivo do álcool, o custo público dos problemas associados direta e indiretamente onera serviços de saúde, relaciona-se à perda de produtividade geral e acarreta gastos com o sistema judiciário e criminal, intervenção social e outros gastos não tangíveis diretamente (Gallassi, Alvarenga, Andrade, \& Couttolenc, 2008).

Por meio do II Levantamento Domiciliar sobre o Uso de Drogas Psicotrópicas no Brasil, realizado nas 108 cidades com mais de cem mil habitantes, em 2005, estimou-se que o álcool foi a substância mais consumida no país, com prevalência de uso na vida de $74,6 \%$ e uma prevalência estimada de $12,3 \%$ de dependentes (Carlini \& Galduróz, 2007). Ademais, segundo o I Levantamento Nacional dos Padrões de Consumo de Álcool do Brasil, estimou-se que entre os adultos, $52 \%$ beberam pelo menos uma vez ao ano. Desses, $60 \%$ dos homens e $33 \%$ das mulheres consumiram 5 doses ou mais, um padrão de consumo pesado que já pode ser considerado como abusivo, sendo um fator de risco para problemas de saúde e de outras ordens (Laranjeira, Pinsky, Zaleski, \& Caetano, 2007).

O conjunto de problemas que o consumo abusivo de álcool pode acarretar, tanto em termos individuais quanto em termos de saúde pública, bem como a possibilidade de redução destes problemas, justifica a busca por intervenções mais compreensivas e efetivas para lidar com o problema (Abou-Saleh, 2006). Um dos aspectos relacionados à efetividade destas intervenções está ligado à forma como os profissionais envolvidos no processo 
percebem, acolhem e lidam com tais usuários, pois, independente do nível de atenção à saúde, será exigido dos profissionais uma adequação de cada intervenção, além do domínio de técnicas específicas e habilidades pessoais para a compreensão e manejo efetivo de uma situação envolvendo problemas com o consumo de álcool (Abou-Saleh, 2006).

Segundo Corrigan (2004), muitas pessoas que poderiam se beneficiar de serviços de saúde, principalmente com serviços de saúde mental, escolhem não buscá-los ou interrompem o tratamento prematuramente, ainda que se ofereça uma intervenção com um modelo adequado. Uma das hipóteses que possuem evidências empíricas para explicar essa baixa aderência ao tratamento aponta para o processo de estigmatização dos usuários como fator que contribui para que estes pacientes não busquem ou desistam do tratamento. Ainda neste contexto, o processo de estigmatização é apontado como um fator que pode afetar negativamente a auto-estima do usuário, como também reduzir suas possibilidades de inserção na sociedade (Corrigan, 2004; Dovidio, Major, \& Crocker, 2003). Nesse sentido, a estigmatização dos usuários de álcool torna-se um aspecto relevante para melhor compreensão das estratégias de intervenção voltadas para essa população.

A partir de uma perspectiva psicossocial, tal processo de estigmatização é compreendido como dinâmico e contextual, produzido socialmente, moldado por forças históricas e sociais, tendo como características fundamentais o reconhecimento da diferença com base em alguma característica distinguível ou "marca" e uma conseqüente desvalorização do seu portador (Dovidio et al., 2003). Dentro desta perspectiva teórica, torna-se também importante definir os aspectos relativos aos processos de percepção e processamento das informações sociais e sua relação nos âmbitos cognitivos, afetivos e comportamentais (Hinshaw, 2007). Dessa forma, os estereótipos e a atribuição de causalidade tornam-se conceitos-chave dentro desta perspectiva teórica, já que possibilitam a construção de categorias de análise e mensuração úteis na sistematização, operacionalização e compreensão do comportamento social relacionado ao estigma (Corrigan, 2004; Michener, DeLamater, \& Myers, 2003; Palm, 2006).

Os estereótipos podem ser definidos como crenças acerca de características, atributos e comportamentos de um determinado grupo, e teorias de como e porque certos atributos estão relacionados (Hilton \& von Hippel, 1996). Além disso, eles possibilitam categorizar rapidamente os indivíduos como pertencentes a um grupo, unicamente com base em informações simples e acessíveis que, por si só, permitiriam classificálos como membros de um grupo. Tal simplificação reduz a complexidade das interações sociais auxiliando nos processos de formação de impressão e previsão de comportamento (Michener et al., 2003; Rodrigues, Jablonski, \& Assmar, 2002).

Apesar dos estereótipos fornecerem algumas informações úteis acerca dos grupos, tais informações acabam por ser super generalizadas, dando margem à caracterização de todos os indivíduos de um grupo em função unicamente de atributos tidos como típicos do grupo, a despeito das diferenças individuais. Uma das hipóteses explicativas desse fenômeno baseia-se na idéia básica de que o sistema perceptivo humano é ativo no processo de seleção dos estímulos relevantes no ambiente, e que por economia psíquica, formaria perceptos de maneira bastante simplificada e com o mínimo de atenção e esforço possível dos objetos sociais percebidos. A força e a inevitabilidade dos estereótipos não seriam atenuáveis, contudo, a aplicação dos estereótipos poderia ser reduzida através da sua concorrência com moderadores sócio-cognitivos que inibem a aplicação direta desses estereótipos, evitando situações de discriminação automática. Apesar de as pessoas poderem ter estereótipos de algo, podem não estar totalmente de acordo com ele e nem ao menos perpetuar ações discriminatórias (Lima \& Vala, 2004). Contudo, tais formas de seleção, categorização e simplificação da informação social, quando em conjunto a fracos moderadores sócio-cognitivos, poderiam influenciar as visões sobre diversas questões sociais e de saúde. Entre estas questões, HIV/AIDS, obesidade, crime e violência, e mesmo o alcoolismo, exercem um papel importante no processo de categorização que constitui uma das bases do processo de estigmatização (Corrigan, Markowitz, Watson, Rowan, \& Kubiak, 2003).

A atribuição de causalidade, que também faz parte do processo de percepção social e tem um papel importante na estigmatização, relaciona-se diretamente à possibilidade de se atribuir determinada causa a um comportamento individual, com base nas informações obtidas através da observação deste comportamento (Michener et al., 2003). As atribuições de causalidade poderiam ser direcionadas para dois pólos explicativos. O primeiro estaria ligado às atribuições de causalidade relacionadas a um fator situacional, que pode ser entendido como fatores externos ao controle individual que se manifestam no ambiente e ocasionam um comportamento. A segunda forma surge através da atribuição de causa de um comportamento como fruto de disposições individuais, como estados internos (afetivos, volitivos e cognições) ou características individuais como fatores de personalidade e de caráter que são compreendidos como capazes de direcionar e controlar um comportamento (Michener et al., 2003).

Algumas condições de saúde são comumente percebidas através de modelos de atribuição de causalidade disposicional, caracterizados principalmente através da identificação de uma fraqueza pessoal e/ou problemas morais ligados a um caráter deteriorado que está associado diretamente ao surgimento da condição e sua possibilidade de cura. Portanto, a principal característica de uma atribuição moral representa o envolvimento de uma responsabilização do indivíduo, conseqüente de uma fraqueza de caráter (Palm, 2006). Notadamente, esse tipo de atribuição está associado com condições de saúde que são extremamente estigmatizadas, tais como a AIDS e transtornos relacionados ao consumo de álcool e outras drogas, os quais são apontados por diversos autores como as condições de saúde mais moralizadas e estigmatizadas em todo o mundo (Corrigan et al., 2005; Palm, 2006).

Nesse contexto, o indivíduo, ao ser estereotipado como alcoolista, a despeito da funcionalidade diagnóstica para o seu tratamento, passa a ser rotulado e pode ter sua identidade social deteriorada. $\mathrm{O}$ surgimento de tal problema passa a ser 
concebido como um problema de fundo moral e, por conseguinte, é compreendido como de foro exclusivamente individual, cuja responsabilidade pela solução do uso problemático deveria partir de uma motivação interna do paciente. Essa concepção moralizante do problema justifica a desresponsabilização de outras pessoas envolvidas no tratamento, tais como a família e os profissionais, contribuindo para um prejuízo no tratamento e estigmatização do usuário (Palm, 2006).

O processo de estigmatização e os seus diversos impactos estão rotineiramente associados com a dependência de álcool e outras drogas, sendo notadamente uma das principais condições de saúde que carregam uma conotação moralizante, sendo afetada pela estigmatização (Corrigan et al., 2005; Palm, 2006). A estigmatização e culpabilização dos usuários por parte da população, e em especial por parte dos profissionais de saúde, podem criar barreiras na qualidade do cuidado em saúde dificultando o acesso, prejudicando o tratamento e restringindo as chances de inserção social destes usuários.

Estudos confirmam a hipótese de que o uso de álcool e de outras drogas é um comportamento visto negativamente por profissionais de saúde (Ronzani, Furtado \& Higgins-Biddle, 2009; Silveira, Martins \& Ronzani, 2009) e até mesmo por estudantes da área (Abarca \& Pillon, 2008; Lopes \& Luis, 2005). Desta forma, uma das estratégias alternativas à concepção moralizante do usuário é o foco na mudança de atitudes dos profissionais de saúde como forma de prevenir os danos associados à estigmatização, contribuindo para melhoria de índices de adesão e de eficácia do tratamento (Ritsher \& Phelan, 2004). Essas ações são planejadas partindo-se do princípio de que a automaticidade e estereotipização podem ser controladas ou modificadas por mediadores sócio-cognitivos (Lima \& Vala, 2004), sendo que um dos contextos estratégicos para se estudar e trabalhar atitudes, tais como as envolvidas no processo de estigmatização, seria na formação dos futuros profissionais de saúde (Ronzani, 2007).

Conhecer as concepções sobre o uso de álcool entre estudantes dos cursos ligados à saúde e possíveis processos decorrentes relacionados à estereotipização e moralização por parte dos estudantes em relação a esses usuários, torna-se um ponto relevante, à medida que essa primeira avaliação pode fornecer bases para melhorias curriculares dos cursos em relação ao conhecimento sobre o abuso de substâncias psicoativas e, conseqüentemente, melhor formação do acadêmico. Este processo implica o estabelecimento de estratégias adequadas de mudança de atitudes desses futuros profissionais visando a uma melhoria da qualidade no atendimento aos usuários de álcool.

O objetivo do presente estudo foi investigar os estereótipos e a atribuição moral de estudantes dos cursos de graduação da área de saúde dos cursos de Enfermagem, Medicina, Psicologia e Serviço Social que estavam próximos de realizar seus estágios profissionais curriculares. A principal hipótese deste estudo foi de que, apesar do contexto privilegiado de ensino de uma universidade e do estágio avançado em que os estudantes estão no curso, a dependência de álcool seria uma das condições de saúde mais moralizadas. Além disso, esperava-se que as dificuldades pessoais para lidar com pacientes alcoolistas fossem maiores do que outros grupos de pacientes e estivessem relacionadas com o modelo de percepção dos alcoolistas e estereotipização entre os estudantes.

\section{Método}

\section{Participantes}

Participaram da pesquisa 146 estudantes dos cursos de saúde de uma universidade federal, distribuídos entre Enfermagem (30,1\%), Medicina (22,6\%), Serviço Social (24,0\%) e Psicologia $(23,3 \%)$. A maioria dos estudantes era do gênero feminino $(81,9 \%)$. Com relação à faixa etária, esta variou de 19 anos até 41 anos, a idade média foi de 22,3 anos $(D P=2,4)$ e a idade mediana de 22 anos, sendo que $97,1 \%$ eram solteiros.

\section{Procedimentos}

O estudo aconteceu entre os meses de março e maio de 2008. Após a aprovação da pesquisa pelos coordenadores de curso ou diretores das faculdades e submissão ao comitê de ética em pesquisa da instituição, foi feito um agendamento com os professores para marcação da coleta de dados entre os estudantes. Logo após a apresentação da pesquisa e esclarecimento acerca dos aspectos éticos envolvidos, os estudantes eram convidados a ler e assinar o Termo de Consentimento Livre e Esclarecido para participar do estudo. Tais procedimentos foram consubstanciados através do parecer do Comitê de Ética em Pesquisa da instituição (n1160/2007).

\section{Delineamentos da Pesquisa}

O presente estudo foi do tipo exploratório, não controlado, de definição intencional da amostra. A escolha das turmas que compuseram a amostra foi feita seguindo um critério de conveniência que buscou uma maior homogeneidade entre os participantes ao adotar o parâmetro de seleção mediante a organização pedagógica dos cursos, seguindo os seguintes critérios de seleção para sua inclusão no estudo: (1) não ter cursado o estágio obrigatório do curso; e (2) estar matriculado no período mais próximo de poder iniciar o estágio curricular obrigatório da instituição. Adotando tais critérios, oito participantes não foram incluídos nas análises. Para melhor operacionalização das hipóteses de estudo e obtenção de níveis de mensuração mais objetivos, optou-se por definir quatro aspectos principais a serem avaliados no presente estudo: (1) Estereótipos acerca da dependência do uso álcool; (2) Modelo de atribuição de responsabilidade por essa condição, (3) Dificuldade pessoal do estudante em lidar com dependentes de álcool; e (4) Informações recebidas pelos estudantes durante a graduação em relação aos efeitos do uso de álcool.

\section{Instrumentos}

Foram utilizados como instrumentos para coleta de dados, questionários auto-aplicáveis, administrados em grupo, durante o horário de aula. Buscou-se intervir o mínimo possível na rotina dos estudantes e manteve-se o controle necessário para a padronização da aplicação. Os seguintes instrumentos foram utilizados:

Questionário Sobre Características dos Estudantes. 
Objetivou caracterizar os participantes da pesquisa através de dados sócio-demográficos, como escolaridade, gênero, estado civil e se já tiveram algum tipo de experiência profissional em serviços de saúde. Além da motivação para realizar atividades de prevenção em relação ao uso abusivo de álcool, avaliada em uma escala do tipo Likert, que variava de 1 (totalmente desmotivado), passando pelo 3 (neutro), até 5 (totalmente motivado).

Escala de Moralização Sobre Uso de Álcool. AAvaliação da moralização do uso de álcool foi feita através de uma escala de estereótipos que relacionava características como falta de força de vontade, fraqueza de caráter e problemas morais ao grupo de alcoolistas. A Escala de Moralização sobre o uso de álcool foi derivada da Escala Marcus, desenvolvida para avaliar crenças e estereótipos sobre usuários de álcool. Dos itens originais da escala, foram utilizados 23 itens retirados do trabalho de Babor et al. (1986). A partir da utilização de tais itens em um estudo mais amplo com profissionais de Atenção Primária à Saúde (Ronzani, Furtado \& Higgins Biddle, 2009) foi realizado o procedimento de Análise Fatorial para o agrupamento de itens por fatores. A escala de moralização foi então definida com cinco itens com proposições estereotípicas do tipo "Alcoolismo é sinal de fraqueza de caráter" e respostas do tipo Likert, que variavam de 1 (discordo totalmente) a 7 (concordo totalmente). A escala apresentava variação de pontuação de 5 a 35 . Na avaliação de fidedignidade da escala, procedeu-se o teste de fidedignidade de Alfa de Cronbach e observou-se o valor de 0,77 na correlação entre os itens. Os itens da escala são os seguintes: (1)Alcoolismo é sinal de fraqueza de caráter; (2) Alcoólatras não se preocupam com seus problemas; (3) Alcoólatras são pessoas moralmente fracas; (4) Alcoólatras são pessoas sem determinação; (5) Alcoólatras não querem parar de beber.

Questionário sobre Modelo de Percepção sobre Problemas de Saúde. Para avaliar o modelo de percepção dos estudantes sobre os usuários de álcool, utilizou-se o modelo de percepção de Brickman apresentado por Palm (2006), no qual se define quatro modelos ideais, baseados na atribuição de responsabilidade pelo surgimento e solução de uma condição de saúde. Esses modelos são os seguintes: Modelo Moral - alta responsabilidade pelo aparecimento e alta responsabilidade pela solução; Modelo Médico - baixa responsabilidade pelo aparecimento e baixa responsabilidade pela solução; Modelo Compensatório - baixa responsabilidade pelo aparecimento e alta pela solução; Modelo Iluminado - alta responsabilidade pelo aparecimento e baixa responsabilidade pela solução.

Cada um destes modelos é apresentado com algumas de suas principais características em relação à dependência de álcool, conforme descrito por Palm (2006): (a) Modelo Moral: a responsabilidade pelo aparecimento e solução de determinado problema de saúde é atribuída ao próprio indivíduo. $\mathrm{O}$ alcoolismo é visto como mau hábito, a causa do alcoolismo advém de uma fraqueza moral e falha por resolver seus problemas, e poderia ser atribuída à falta de força de vontade; (b) Modelo Médico: o indivíduo é minimamente responsável pela causa do problema porque o alcoolismo tem uma etiologia biológica. Sendo assim, a responsabilidade individual por resolver e controlar seu problema é mínima. $\mathrm{O}$ alcoolismo é visto como uma doença irreversível e sem cura. $\mathrm{O}$ tratamento para o problema seria possível através do acompanhamento de um profissional ou programa que auxilie na busca da abstinência; (c) Modelo Compensatório: a responsabilidade individual pelo surgimento ou causa do problema é mínima. Entretanto, o indivíduo é responsável por resolver seu problema. Portanto, a metodologia de tratamento articula a conjunção da perspectiva de doença e a abordagem de solução de problemas. O paciente não é culpado diretamente pelo seu problema, mas responsável por lidar com ele desenvolvendo habilidades de enfrentamento ou adquirindo um melhor suporte social; (d) Modelo Iluminado: os indivíduos são responsáveis por causar seu problema, porque eles se levaram pelo impulso de beber e perder o controle. Entretanto, os alcoolistas têm um mínimo controle por resolver seu problema. Sendo assim, o tratamento necessita que o alcoolista admita a fraqueza perante a bebida, assim como submeta o seu controle a uma Força Maior que ilumine seus passos. Essa força poderia ser a comunidade ou outros alcoolistas que estão se recuperando.

O questionário utilizado para construir o modelo foi composto por uma pergunta acerca da responsabilidade dos pacientes com relação ao aparecimento de um problema de saúde e outra sobre a responsabilidade que esse paciente teria para solucionar esse problema. A possibilidade de resposta de concordância numa escala de um a cinco, onde um corresponde a "nada responsável" e cinco a "totalmente responsável", foi oferecida para as perguntas: "O quanto você acha que o paciente é responsável pelo aparecimento/evolução dos seus problemas de saúde?"; e, "O quanto você acha que o paciente é responsável pela solução dos seus problemas de saúde?". Para a avaliação do modelo entre os estudantes as respostas de cada participante foram recodificadas retirando-se o neutro da escala e agrupando as respostas entre baixa e alta atribuição para responsabilidade, tanto com relação à percepção acerca da solução do problema, quanto para o aparecimento deste. Foi também utilizado o modelo gráfico para comparação entre as doenças e os agravos, onde as medianas das respostas das escalas para cada agravo e doença foram transpostas para um eixo cartesiano (x,y), sendo o eixo das abscissas $(\mathrm{x})$ relativo ao aparecimento e evolução e o eixo das ordenadas $(\mathrm{y})$ à responsabilidade de solução. $\mathrm{O}$ ponto médio (3) da escala foi definido como origem do eixo. Desta forma, as medianas menores que três foram consideradas baixas e as maiores que três consideradas altas.

Avaliação de Dificuldade Pessoal. A avaliação da dificuldade para lidar com pacientes dependentes do uso de álcool foi feita através da comparação desta condição de saúde com a hipertensão arterial. Utilizou-se como parâmetro de dificuldade a hipertensão arterial, por considerar que procedimentos de prevenção e tratamento deste problema estarão incluídos na rotina de trabalho desses futuros profissionais, considerando-a como um parâmetro neutro de comparação. As respostas foram do tipo Likert, que variaram de -3 (menor grau de dificuldade), passando pelo 0 (equivalendo a grau semelhante de dificuldade pessoal ao lidar com hipertensos) até +3 (maior grau de dificuldade). As respostas foram posteriormente convertidas em números absolutos de um a sete para classificar a dificuldade dos futuros profissionais em lidar com os alcoolistas. Com a 
finalidade de complementar essa mensuração outras condições de saúde foram também avaliadas.

Avaliação das informações recebidas sobre os efeitos do uso de álcool. A avaliação das informações recebidas durante o curso de graduação sobre os efeitos do uso de álcool foi feita através de uma escala do tipo Likert, variando de 1 - "Não recebi informações" a 4 - "Recebi muitas informações". Foi também adicionada uma questão para avaliar como os estudantes consideravam as informações recebidas: "suficientes", "insuficientes", ou se "não receberam informações".

Análise dos Dados. Os dados foram primeiramente digitados no software estatístico SPSS versão 15.0, com a finalidade de corrigir eventuais erros de digitação e garantir a qualidade dos dados. Posteriormente os dados foram organizados e analisados estatisticamente. Inicialmente foram utilizadas técnicas descritivas para apresentar as freqüências $(n)$, porcentagens $(\%)$, média $(M)$, mediana (P50), desvio padrão $(D P)$, valores mínimos e máximos. A partir de então, verificou-se através do teste estatístico de Kolmogorov-Smirnov a hipótese nula de não existência de diferença estatística entre a distribuição amostral das variáveis numéricas escalares utilizadas neste estudo com uma distribuição normal. A hipótese nula foi rejeitada para todas as variáveis testadas ( $\operatorname{com} p<0,05$, no teste KolmogorovSmirnov), portanto, optou-se por prosseguir as análises inferenciais através de estatísticas não-paramétricas, indicando a mediana como medida de tendência central mais adequada das variáveis. O teste de Mann-Whitney foi utilizado para testar a hipótese nula da não existência de diferença estatisticamente significativa entre a média dos postos das variáveis numéricas escalares de duas amostras independentes; quando essa comparação era feita entre mais de duas amostras independentes foi utilizado o teste de Kruskal-Wallis. Utilizou-se a correlação bivariada de Spearman para a análise de existência de covariação entre duas variáveis numéricas escalares analisando a existência de relacionamento estatisticamente significativo e o coeficiente de correlação entre as variáveis ( $\rho$ de Spearman). O teste do Qui-quadrado de Pearson foi utilizado para testar a hipótese nula de não existência de diferença estatística entre a proporção da distribuição de grupos definidos a partir de variáveis nominais relacionadas. $\mathrm{O}$ teste exato de Fisher foi calculado para obter valores de significância corrigidos quando violadas as propriedades de número mínimo de observações por célula no teste do Qui-Quadrado. Adotou-se para todas as análises inferenciais o nível de significância de $95 \%(p<0,05)$.

\section{Resultados}

\section{Descrição sócio-demográfica e características dos estudantes}

Entre os estudantes, 51,4\% afirmaram que já tiveram algum tipo de experiência profissional em serviços de saúde, sendo que essas atividades caracterizavam-se por estágios extra-curriculares em 39,9\% dos casos, 3,6\% já haviam feito estágio básico, 2,9\% dos casos já tiveram algum tipo de emprego formal na área de saúde e o restante não informou que tipo de experiência já tiveram. No curso de Medicina, especificamente, $93,5 \%$ dos estudantes afirmaram já ter realizado algum tipo de estágio extra-curricular, em contraste com $6,2 \%$ dos estudantes do curso de Serviço Social.

Para $81,5 \%$ dos estudantes, a identificação com o curso foi o principal motivo de escolha para seguir na profissão, sendo que $70,0 \%$ relataram estarem satisfeitos com o curso e $80,4 \%$ disseram estar satisfeitos ou totalmente satisfeitos com seu desempenho acadêmico. Quando indagados sobre o quanto estão envolvidos em atividades de promoção e prevenção de saúde, $27,8 \%$ responderam nunca ou raramente, $30,7 \%$ declararam às vezes, $41,6 \%$ sempre ou quase sempre. A respeito da motivação para realizar atividades de prevenção do uso abusivo de álcool, uma minoria relatou estar desmotivado ou totalmente desmotivado $(6,5 \%)$, a maior parte estava motivada de alguma forma $(55,1 \%)$, seguidos por neutros $(38,4 \%)$, um participante não respondeu essa questão. A porcentagem de estudantes que afirmaram ter algum familiar usuário pesado ou dependente de álcool foi de $35,8 \%$. Possuir alguém na família que seja usuário pesado ou dependente de álcool não foi um fator relacionado com a motivação do estudante para realizar trabalhos de prevenção ao uso nocivo de álcool no futuro ( $p=0,63$, no teste exato de Fisher). $\mathrm{O}$ envolvimento do estudante em atividades de prevenção de doenças e promoção de saúde apresentou uma fraca correlação ( $\rho=0,29, p \leq 0,001)$ com a motivação para realizar trabalhos de prevenção ao uso nocivo de álcool no futuro.

\section{Moralização do uso de álcool}

O grupo de estudantes apresentou um escore mediano de 11 pontos na escala de moralização, a amplitude da escala variou de 5 até 35 . A porcentagem média de concordância desta escala foi de 32,34\%, levando em consideração o escore total máximo de concordância com as assertivas estereotípicas da escala escore total de 35 pontos. Não houve diferença entre gênero na moralização de alcoolistas, assim como ter alguém na família que seja usuário pesado ou dependente de álcool. Ao se analisar especificamente a moralização entre os cursos, observou-se que não houve uma diferença estatisticamente significativa nesta comparação. Foi realizada uma análise de correlação entre a escala de moralização do uso de álcool com a dificuldade relatada para lidar com pacientes alcoolistas, porém não foi observada uma correlação estatisticamente significativa. Diferente do esperado, não se observou diferença estatística significativa entre a mediana dos postos dos modelos de percepção para alcoolismo em relação à escala de moralização.

\section{Modelos de percepção de doenças e agravos}

Não foi encontrada diferença estatística significativa entre os cursos, para a média dos postos das respostas relacionadas à responsabilidade pelo surgimento e responsabilidade pela solução entre as condições. Portanto, optou-se por analisar os modelos de percepção sem fazer diferenciação entre os cursos.

A análise da freqüência das classificações do modelo de percepção para cada doença e agravo está descrita na Tabela 1. Para todas as condições de saúde, exceto para a depressão ( $p=0,43$ no Qui-Quadrado de Pearson), houve uma diferença significativa na distribuição de freqüência entre os modelos 
( $p=0,002$ no teste de Qui-Quadrado de Pearson). As condições de saúde que mais se enquadraram no modelo moral foram: Dependência Maconha/Cocaína (94,4\%), Tabagismo (93,2\%), Alcoolismo (91,8\%), Obesidade (85,3\%), HIV/AIDS (39,5\%), Depressão $(40,4 \%)$, Diabetes $(20,8 \%)$, Hanseníase $(1,4 \%)$ e Esquizofrenia $(1,2 \%)$. O modelo moral foi predominante para as cinco primeiras condições, enquanto o modelo iluminado para HIV/AIDS (51,9\%), modelo compensatório para diabetes $(56,6 \%)$ e o modelo médico para esquizofrenia $(88,1 \%)$ e hanseníase $(55,1 \%)$.

A partir da análise do modelo gráfico de Brickman observou-se que as condições classificadas através do modelo

Tabela 1

Distribuição de Freqüência e Porcentagem sobre a Percepção de Condições de Saúde entre os Estudantes com Modelo Brickman

\begin{tabular}{|c|c|c|c|c|}
\hline & \multicolumn{4}{|c|}{ Modelo de Percepção Brickman } \\
\hline Condição de Saúde & $\begin{array}{l}\text { Moral } \\
n(\%) \\
\end{array}$ & $\begin{array}{l}\text { Médico } \\
n(\%) \\
\end{array}$ & $\begin{array}{c}\text { Iluminado } \\
n(\%)\end{array}$ & $\begin{array}{c}\text { Compensatório } \\
n(\%)\end{array}$ \\
\hline Dependência Maconha/Cocaína* & $101(94,4 \%)$ & $2(1,9 \%)$ & $2(1,9 \%)$ & $2(1,9 \%)$ \\
\hline Tabagismo* & $96(93,2 \%)$ & $1(1,0 \%)$ & $0(0,0 \%)$ & $6(5,8 \%)$ \\
\hline Alcoolismo * & $90(91,8 \%)$ & $14(9,9 \%)$ & $9(6,4 \%)$ & $5(3,5 \%)$ \\
\hline Obesidade* & $58(85,3 \%)$ & $1(1,5 \%)$ & $1(1,5 \%)$ & $8(11,8 \%)$ \\
\hline Depressão $* *$ & $23(40,4 \%)$ & $15(26,3 \%)$ & $0(0,0 \%)$ & $19(33,3 \%)$ \\
\hline HIV/AIDS* & $32(39,5 \%)$ & $6(7,4 \%)$ & $42(51,9 \%)$ & $1(1,2 \%)$ \\
\hline Diabetes* & $11(20,8 \%)$ & $12(22,6 \%)$ & $0(0,0 \%)$ & $30(56,6 \%)$ \\
\hline Hanseníase* & $1(1,4 \%)$ & $38(55,1 \%)$ & $1(1,4 \%)$ & $29(42,0 \%)$ \\
\hline Esquizofrenia* & $1(1,2 \%)$ & $74(88,1 \%)$ & $0(0,0 \%)$ & $9(10,7 \%)$ \\
\hline
\end{tabular}

$* p<0,002$ no teste do Qui-Quadrado de Pearson; $* * p>0,05$ no teste do Qui-Quadrado de Pearson

moral foram: Dependência de maconha/cocaína (solução $\mathrm{P} 50=5$; Aparecimento P50=4), Tabagismo (Solução P50=5; Aparecimento P50=4) e Alcoolismo (Solução P50=4; Aparecimento $\mathrm{P} 50=4)$. A Depressão e Obesidade não foram descriminadas em um único modelo, sendo percebidas entre o modelo compensatório e moral (Solução P50=4; Aparecimento $\mathrm{P} 50=3)$. A Esquizofrenia foi percebida através do modelo Médico (Solução P50=2; Aparecimento P50=1), enquanto a Diabetes no modelo Compensatório (Solução P50=3,5; Aparecimento P50=2,5). O HIV/AIDS não foi discriminado por um único modelo, sendo percebido entre o modelo Moral e Iluminado
(Solução P50=3; Aparecimento P50=4) e a Hanseníase entre o modelo Moral e Médico (Solução P50=3; Aparecimento P50=1). Os resultados obtidos através do método gráfico estiveram em relativa concordância com os resultados do método de classificação individual e serve como um modelo ilustrativo da relação entre atribuição de responsabilidade pela solução e aparecimento das condições, sendo apresentado na Figura 1.

\section{Dificuldades pessoais para lidar com pacientes}

Em relação às dificuldades pessoais para lidar com pacientes com determinados problemas de saúde, aqueles que representaram

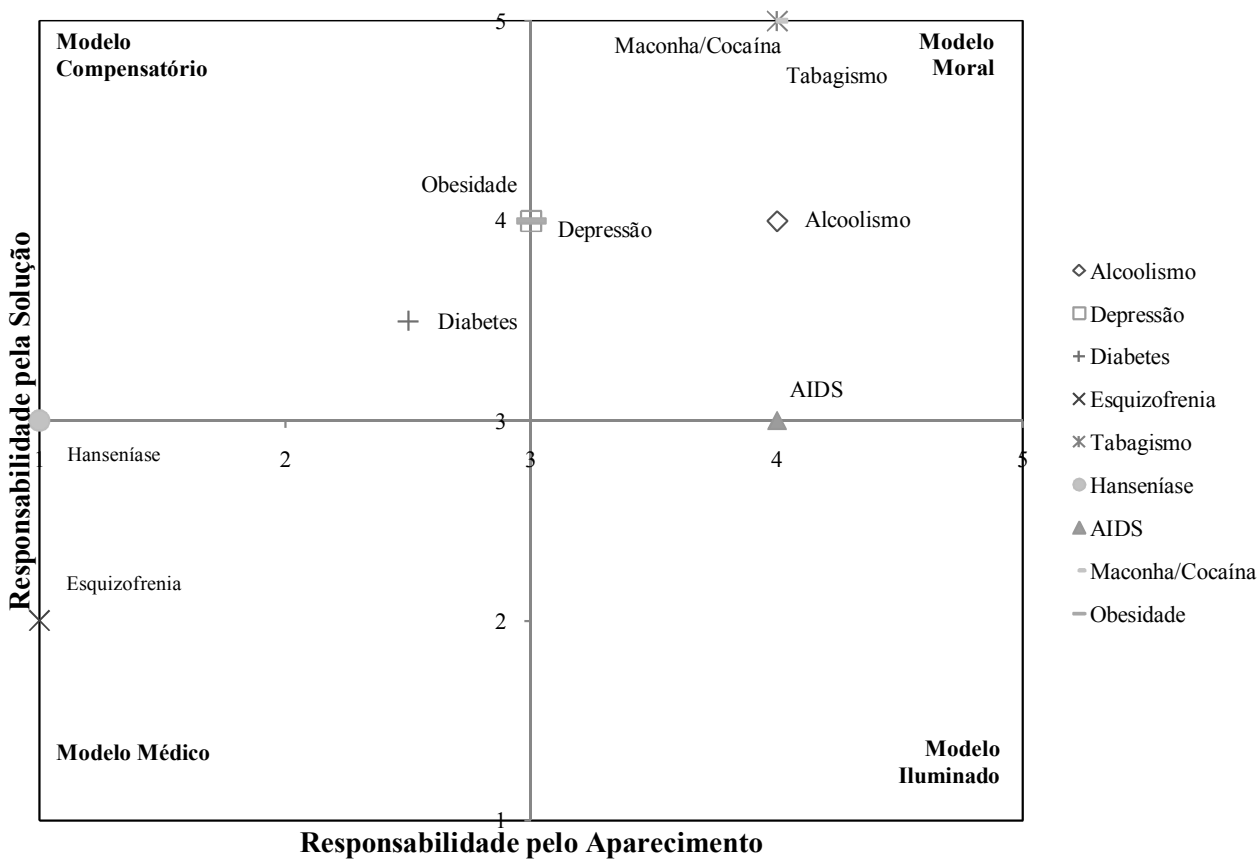

Figura 1. Modelo Gráfico Baseado no Modelo de Percepção de Brickman. 
as maiores dificuldades em relação aos hipertensos, através da análise das medianas foram, respectivamente, os dependentes de maconha/cocaína $(P 50=6)$, esquizofrênicos $(P 50=6)$, alcoolistas ( $\mathrm{P} 50=6)$, depressivos ( $\mathrm{P} 50=5), \mathrm{HIV}(\mathrm{P} 50=5)$, fumantes ( $\mathrm{P} 50=5)$, hansenianos $(\mathrm{P} 50=4)$, obesos $(\mathrm{P} 50=4)$ e diabéticos $(\mathrm{P} 50=4)$. Não foram observadas diferenças significativas na dificuldade de lidar com pacientes com as condições de saúde estudadas em relação ao gênero e curso dos estudantes e presença de usuário pesado ou dependente de álcool na família.

\section{Informações recebidas durante o curso sobre o uso de álcool}

Quando avaliadas as informações recebidas durante o curso sobre os efeitos do uso de álcool, a maioria dos estudantes $(44,4 \%)$ afirmou ter recebido algumas informações, $38,5 \%$ recebeu muito poucas ou nenhuma informação e $17 \%$ receberam muitas informações, sendo que 57,8\% consideraram as informações recebidas como insuficientes. Não foi possível calcular a existência de diferença entre os conhecimentos recebidos e outras variáveis como curso, motivação para atividades de prevenção devido à baixa freqüência entre as células da tabela cruzada entre as duas variáveis. Não foram encontradas diferenças estatísticas entre os conhecimentos e dificuldade para lidar com alcoolistas, responsabilidade pelo surgimento ou solução do problema com álcool conhecimento.

\section{Discussão}

A hipótese de que a dependência de álcool seria uma das condições mais moralizadas pelos estudantes foi corroborada neste estudo. O tabagismo e a dependência de maconha e cocaína também foram percebidos de forma semelhante, o que segue em consonância com os outros estudos que apontam para evidências de que o uso de álcool e outras drogas é uma das condições mais moralizadas, quando comparadas a outros problemas de saúde (Corrigan et al., 2005; Palm, 2006).

A ausência de diferença entre os cursos para os modelos de percepção, tanto no quesito moralização, quanto nos outros modelos, pode indicar a ausência de uma abordagem da temática álcool e outras drogas, nesses períodos dos cursos. Existindo já uma abordagem do tema, pode-se inferir que os conhecimentos passados acerca da dependência seguiram um modelo moral que poderá reduzir a capacidade da oferta de um cuidado de qualidade aos dependentes. Prislin, Shultz e Singleton (1999) avaliaram a concepção de estudantes do curso de Medicina sobre dependentes químicos através de um questionário antes e depois da realização de um mini-curso e concluíram que após o curso, os estudantes fizeram menos julgamentos morais sobre a presença de fraqueza de caráter em pacientes com abuso de álcool. Percebe-se, portanto, que a consideração das crenças e atitudes acerca de problemas de saúde e agravos se faz fundamental para o desenvolvimento de métodos de ensino mais eficazes que possam assegurar a formação de um profissional melhor capacitado para lidar com esse tipo de paciente e com uma postura menos moralizante (Ronzani, 2007).

Como já exposto, um dos critérios de inclusão da amostra do presente estudo foi selecionar os alunos que estavam matriculados no período mais próximo de iniciarem seus estágios curriculares, ou seja, o fato de ainda não terem iniciado o contato direto com os usuários da rede de saúde pode justificar a alta moralização dos dependentes de álcool por esses estudantes. Considerando a moralização de um problema de saúde como a atribuição de responsabilidade ao indivíduo pelo surgimento do problema e a solução deste, é mais provável que um grupo moralizado seja evitado em seu convívio social ou mesmo não receba ajuda quando necessário (Corrigan et al., 2003; Corrigan et al., 2005). De acordo com Volmer, Maesalu e Bell (2008), é de fundamental importância que as faculdades criem possibilidades de contato entre estudantes e usuários dos serviços de saúde já nos primeiros anos dos cursos, visto que a promoção de familiaridade e contato entre pacientes e futuros profissionais pode ser um mecanismo eficaz em formar atitudes mais favoráveis em relação ao tratamento do paciente (Volmer et al., 2008).

Doenças não controláveis, como o câncer e problemas do coração, despertam sentimentos como compaixão e aumentam a disposição para ajudar (Corrigan et al., 2003); a dependência de álcool e de outras drogas, as quais são percebidas como controláveis, seguem um modelo moralizado, o que pode ter um impacto negativo na própria disposição dos estudantes para ajudar estes pacientes. Neste caso, a moralização do consumo de álcool por parte dos estudantes pode ter importantes conseqüências para estes futuros profissionais de saúde, quando se defrontarem com este problema em sua prática profissional (Corrigan et al., 2005; Palm, 2006), podendo até mesmo influenciar negativamente os cuidados ofertados aos pacientes (Kelleher, 2007).

A não existência de relacionamento entre moralização de alcoolistas a partir da escala de estereótipos e o modelo moral indica que os grupos de estudantes classificados como aqueles que possuíam outros modelos de atribuição, que não o modelo moral, apresentaram estereótipos sobre alcoolistas semelhantes aos participantes deste modelo. Esse resultado poderia ser explicado a partir da teoria dos estereótipos que os definem como crenças culturalmente compartilhadas, que em determinados grupos podem apresentar uma uniformidade (Michener et al., 2003).

Em concordância com o que era esperado, o grupo de alcoolistas e dependentes de maconha e cocaína foi considerado pelos estudantes como os pacientes mais difíceis para se lidar quando comparados aos hipertensos. Diferente do que era esperado, não houve uma correlação entre dificuldade pessoal e a escala de moralização sobre alcoolistas e os modelos de percepção para cada problema. Assim como no estudo de Volmer et al. (2008), onde gênero e experiência familiar não apresentaram diferença significativa em relação às atitudes dos estudantes de Farmácia com o doente mental, não houve diferença estatística entre dificuldade pessoal para lidar com determinado agravo e o fato de ter um familiar com problemas com álcool ou outras drogas, nem tampouco com o gênero ou o curso dos participantes. O não relacionamento pode ser atribuído a uma avaliação por parte dos estudantes muito mais pautada por uma dificuldade técnica do que uma dificuldade baseada na aceitação do problema. Tal contradição pode também estar relacionada à forma como tais questões foram mensuradas, 
representando uma limitação do estudo, diante da possibilidade de construir inferências mais significativas.

A presente pesquisa tem como limitações adicionais o fato do estudo ter sido conduzido por auto-relato, não avaliando, de fato, tais atitudes e comportamentos que podem ser relatadas de maneira diferente devido a fatores como a desejabilidade social. Outro aspecto a ser ressaltado foi que a amostra se constituiu por uma população muito específica, definida por conveniência, dificultando a generalização dos resultados, permitindo que os dados sejam analisados de forma mais descritivos e com cautela. Entretanto, vale ressaltar que outros estudos conduzidos em diferentes culturas e populações apontam na mesma direção e dão suporte às conclusões obtidas (Corrigan et al., 2003; Corrigan et al., 2005).

A formação no ensino superior é um instrumento fundamental para obtenção de mão-de-obra qualificada nos setores da saúde no país. Para tanto, é importante que a formação profissional envolva conteúdos adequados ao contexto de atuação no país, visando à humanização do cuidado para diferentes condições (Barros \& Pillon, 2006). Apesar da configuração epidemiológica do país apontar para a importância dos problemas relacionados ao consumo de álcool (Laranjeira et al., 2007), a falta de conteúdo acerca deste tema relatada pelos alunos de graduação no presente estudo, pode conduzir os estudantes a desenvolverem concepções errôneas sobre os alcoolistas e mesmo dependentes químicos, podendo influenciar sua futura prática profissional (Prislin et al., 1999). Todavia, uma formação técnica, calcada apenas na transmissão de conhecimentos e com poucas práticas reflexivas, seja sobre o álcool ou outras condições de saúde, não seria suficiente para mudar tais concepções, o que torna relevante o incentivo de modalidades educativas, associadas a práticas humanizadas que se distanciem de modelos moralizantes, associando atitudes positivas e otimistas às intervenções e tratamentos (Lopes \& Luis, 2005; Pinikahana, Happell, \& Carta, 2002). Nesse sentido, concluise que, associado ao conhecimento técnico, faz-se necessário enfocar a mudança de crenças e atitudes sobre usuários de álcool e outras drogas com a finalidade de obter melhorias em ações mais compreensivas que visem a implementação de estratégias de prevenção e reabilitação reduzindo a estigmatização dos usuários (Palm, 2006; Ronzani, 2007).

\section{Referências Bibliográficas}

Abarca, A. M., \& Pillon, S. C. (2008). Nursing students' perception regarding predicting factors of drugs use. Revista Latino-Americana de Enfermagem, 16, 607-613

Abou-Saleh, M. T. (2006). Substance use disorders: recent advances in treatment and models of care. Journal of Psychosomatic Research, 61(3), 305-310.

Babor, T. F., Hesselbrock, M., Radouco-Thomas, S., Feguer, L., Ferrant, J.-P., \& Choquette, K. (1986). Concepts of alcoholism among american, frenchcanadian, and french alcoholic. Annals of the New York Academy of Sciences, 472(1), 98-109.

Barros, M. A., \& Pillon, S. C. (2006). Programa Saúde da Família: desafios e potencialidades frente ao uso de drogas. Revista Eletrônica de Enfermagem, 8(1), 144-149.

Carlini, E. A., \& Galduróz, J. C. (2007). II Levantamento domiciliar sobre o uso de drogas psicotrópicas no Brasil: estudo envolvendo as 108 maiores cidades do país. Brasília, DF: Secretaria Nacional Antidrogas.
Corrigan, P. (2004). How stigma interferes with mental health care. American Psychologist, 59(7), 614-625.

Corrigan, P., Lurie, B. D., Goldman, H. H., Slopen, N., Medasani, K., \& Phelan, S. (2005). How adolescents perceive the stigma of mental illness and alcohol abuse. Psychiatric Services, 56, 544-550.

Corrigan, P., Markowitz, F. E., Watson, A., Rowan, D., \& Kubiak, M. A. (2003). An attribution model of public discrimination towards persons with mental illness. Journal of Health and Social Behavior, 44, 162-179.

Dietler, M. (2006). Alcohol: anthropological/archaeological perspectives. Annual Review of Anthropology, 35(1), 229-249.

Dovidio, J. F., Major, B., \& Crocker, J. (2003). Stigma: introduction and overview. In T. F. Heatherton, R. E. Kleck, M. R. Hebl \& J. G. Hull (Orgs.), The social psychology of stigma (pp. 1-28). New York: The Guilford Press.

Gallassi, A. D., Alvarenga, P. G., Andrade, A. G., \& Couttolenc, B. F. (2008). Custos dos problemas causados pelo abuso do álcool. Revista de Psiquiatria Clínica, 35(1), 25-30.

Hilton, J. L., \& von Hippel, W. (1996). Stereotypes. Annual Review of Psychology, $47(1), 237-271$

Hinshaw, S. P. (2007). Stigma of mental illness and agenda for change. New York: Oxford University Press.

Kelleher, S. (2007). Health care professionals' knowledge and attitudes regarding substance use and substance users. Accident and Emergency Nursing, 15, 161-165

Laranjeira, R., Pinsky, I., Zaleski, M., \& Caetano, R. (2007). I Levantamento nacional sobre os padrões de consumo de álcool na população brasileira. Brasília, DF: Secretaria Nacional Antidrogas.

Lima, M. E. O., \& Vala, J. (2004). Serão os estereótipos e os preconceitos inevitáveis? O monstro da automaticidade. In M. E. Pereira \& M. E. O. Lima (Orgs.), Estereótipos, Preconceitos e Discriminação: perspectivas teóricas e metodológicas (pp. 41-68). Salvador: EDUFBA.

Lopes, G. T., \& Luis, M. A. V. (2005). A formação do enfermeiro e o fenômeno das drogas no estado do Rio de Janeiro - Brasil: atitudes e crenças. Revista Latino-Americana de Enfermagem, 13, 872-879.

Michener, H. A., DeLamater, J. D., \& Myers, D. J. (2003). Percepção Social e Cognição. In Psicologia Social (pp. 130-169). São Paulo: Pioneira Thomson Learning Ltda.

Palm, J. (2006). Moral Concerns - treatment staff and user perspectives on alcohol and drug problems. Estocolmo, Suécia: Universidade de Estocolmo.

Pinikahana, J., Happell, B., \& Carta, B. (2002). Mental health professionals attitudes to drugs and substance abuse. Nursing and Health Sciences, 4, 57-62.

Prislin, M., Shultz, G., \& Singleton, V. A. (1999). Improving education about substance abuse. Academic Medicine, 74, 749-750.

Rehm, J., Room, R., Graham, K., Monteiro, M., Gmel, G., \& Sempos, C. T. (2003). The relationship of average volume of alcohol consumption and patterns of drinking to burden of disease: an overview. Addiction, 98(9), 1209-1228.

Ritsher, J. B., \& Phelan, J. C. (2004). Internalized stigma predicts erosion of morale among psychiatric outpatients. Psychiatric Research, 129, 257-265.

Rodrigues, A., Jablonski, B., \& Assmar, E. M. L. (2002). Psicologia Social. Petrópolis: Vozes.

Ronzani, T. M. (2007). A reforma curricular nos cursos de saúde: qual o papel das crenças? Revista Brasileira de Educação Médica, 31, 38-43.

Ronzani, T. M., Furtado, E. F., \& Higgins-Biddle, J. (2009). Stigmatization of alcohol and other drug users by primary care providers in Southeast Brazil. Social Science \& Medicine, 69(7), 1080-1084.

Silveira, P. S., Martins, L. F., Ronzani, T. M. (2009) Moralização sobre o uso de álcool entre agentes comunitários de saúde. Psicologia: Teoria e Prática, $11,62-75$.

Volmer, D., Maesalu, M., \& Bell, S. (2008). Pharmacy students' attitudes toward and professional interactions with people with mental disorders. International Journal of Social Psychiatry, 54(5), 402-413.

World Health Organization. (2007). WHO expert committee on problems related to alcohol consumption: second report. Genebra: World Health Organization. 
Leonardo Fernandes Martins, mestrando em Psicologia pelo Programa de Pós-Graduação em Psicologia da Universidade Federal de Juiz de Fora, é membro do Pólo de Pesquisa em Psicologia Social e Saúde Coletiva da Universidade Federal de Juiz de Fora. Endereço para correspondência: Rua Muriaé, 189/202, Bairro Democrata, Juiz de Fora-MG CEP:36035-250 Telefone: 3214-0243. E-mail: leomartinsif@gmail.com Pollyanna Santos da Silveira, mestre em Psicologia pela UFJF, é membro do Pólo de Pesquisa em Psicologia Social e Saúde Coletiva da Universidade Federal de Juiz de Fora. Rhaisa Gontijo Soares, mestranda em Psicologia pelo Programa de Pós-Graduação em Psicologia da UFJF, é membro do Pólo de Pesquisa em Psicologia Social e Saúde Coletiva da Universidade Federal de Juiz de Fora. Henrique Pinto Gomide, graduando em Psicologia pela Universidade Federal de Juiz de Fora, é membro do Pólo de Pesquisa em Psicologia Social e Saúde Coletiva da Universidade Federal de Juiz de Fora.

Telmo Mota Ronzani, doutor em Psicobiologia pela UNIFESP, é coordenador do Pólo de Pesquisa em Psicologia Social e Saúde Coletiva da Universidade Federal de Juiz de Fora. 